\title{
METAIS PESADOS COMO INDICADORES DE MATERIAIS DE ORIGEM EM UMA TOPOLITOSEQÜÊNCIA DO TRIÂNGULO MINEIRO, ESTADO DE MINAS GERAIS ${ }^{1}$
}

\author{
TEOGENES SENNA DE OLIVEIRA², LIOVANDO MARCIANO DA COSTA ${ }^{3}$, \\ COSME DAMIÃO CRUZ ${ }^{4}$ e HEINRICH ADOLF HORN ${ }^{5}$
}

\begin{abstract}
RESUMO - Objetivou-se neste trabalho determinar os teores de metais pesados em amostras de solos de uma topolitosequiência coletada no Triângulo Mineiro, Estado de Minas Gerais, para testar a hipótese de que tais elementos podem ser utilizados na identificação e separação da influência do material de origem. Os teores de $\mathrm{Cd}, \mathrm{Cr}, \mathrm{Cu}, \mathrm{Fe}, \mathrm{Mn}, \mathrm{Ni}, \mathrm{Pb}$ e $\mathrm{Zn}$ foram determinados por dois procedimentos de ataque ácido: $\mathrm{HNO}_{3} / \mathrm{HClO}_{4}(4: 1)$ e $\mathrm{HF} / \mathrm{HNO}_{3} / \mathrm{HClO}_{4}(20: 6: 3)$, e dosados por espectrofotometria de absorção atômica. Técnicas de análise multivariada foram utilizadas. Os resultados permitem estabelecer que os metais pesados são indicativos do material de origem, podendo ser usados na separação e delimitação de sua área de influência. As técnicas multivariadas adotadas mostraram-se viáveis nesse modelo de estudo quando analisadas conjuntamente.
\end{abstract}

Termos para indexação: material de origem, metais pesados, análise multivariada.

\section{HEAVY METALS AS INDICATORS OF PARENT ROCKS IN A TOPOLITHOSEQUENCE IN TRIÂNGULO MINEIRO, STATE OF MINAS GERAIS, BRAZIL}

\begin{abstract}
The present study had the purpose to determine the contents of heavy metals in soil samples of a topolithosequence collected in the Triângulo Mineiro, State of Minas Gerais, in order to test the hypothesis that these elements could be used in the identification and separation of the influence of the parent material. The contents of $\mathrm{Cd}, \mathrm{Cr}, \mathrm{Cu}, \mathrm{Fe}, \mathrm{Mn}, \mathrm{Ni}, \mathrm{Pb}$ and $\mathrm{Zn}$ were determined through two types of concentrated acid attack: $\mathrm{HNO}_{3} / \mathrm{HClO}_{4}(4: 1)$ and $\mathrm{HF} / \mathrm{HNO}_{3} / \mathrm{HClO}_{4}$ (20:6:3), and dosed by espectrophotometry of atomic absortion. Procedures of multivariated analysis were used for the statistical data evaluation. The results confirm the hypothesis and permitted to conclude that the heavy metals are indicator of the parent material, being able to be used for the separation and delimitation of the area of their influence. The adopted multivariated techniques proved to be viable for this kind of study only when analysed as a whole.
\end{abstract}

Index terms: parent rock, heavy metals, analysis multivariated.

\section{INTRODUÇÃO}

O estudo dos metais pesados, por serem muito estáveis na natureza, conseqüentemente possíveis

\footnotetext{
${ }^{1}$ Aceito para publicação em 20 de novembro de 1998.

${ }^{2}$ Eng. Agr., D.Sc., Prof. Adjunto, Dep. de Ciência do Solo, Universidade Federal do Ceará, Campus do PICI, Bloco 807, CEP 60455-760 Fortaleza, CE. E-mail: teogenes@ufc.br

${ }^{3}$ Eng. Agr., Ph.D., Prof. Titular, Dep. de Solos, Universidade Federal de Viçosa (UFV), CEP 36571-000 Viçosa, MG. Bolsista do CNPq

${ }^{4}$ Eng. Agr., D.Sc., Prof. Adjunto, Dep. de Biologia Geral, UFV. Bolsista do CNPq

${ }^{5}$ Geólogo, Ph.D., Prof. Adjunto, Dep. de Geologia, Centro de Pesquisas Manoel Teixeira da Costa, CEP 31270-901 Belo Horizonte, MG. Bolsista do CNPq.
}

de serem acumulados no solo ou em sistemas biológicos (Bitell \& Miller, 1974; Lagerwerff, 1977), tem nos últimos anos se concentrado na avaliação dos efeitos da acumulação nos organismos nos diferentes ecossistemas, principalmente pela adição antrópica ao meio ambiente, a transferência na cadeia alimentar e os meios alternativos de convivência com os problemas gerados pelos excessos ocorridos. Neste último caso, a destinação adequada dos resíduos sólidos produzidos pelas diferentes atividades urbano-industriais revela-se atualmente como o principal enfoque de grande número de trabalhos desenvolvidos (Gupta \& Stadelmann, 1983; Gambrell et al., 1991; Singh \& Sekhon, 1991; Malmer et al., 1992; Mariño et al., 1992; Shotyk et al., 1992; 
Ciavatta et al., 1993; Taylor et al., 1993; Pascoe et al., 1994).

É consenso na literatura o fato de que os teores desses elementos no solo refletem os teores do material de origem, com exceção dos casos de intensa deposição por uma fonte antrópica, podendo ser indicativo dos diferentes tipos de materiais de origem e, até, em alguns casos, utilizados em estudos sistemáticos e pedogenéticos de solos, mesmo em condições severas de intemperismo (pedogênese), como as dos trópicos (Mitchell, 1964; Krauskopf, 1972; Valadares, 1975; Valadares \& Catani, 1975; Tiller, 1980; Curi, 1983; Chittleborough et al., 1984; Moura, 1985; Resende et al., 1986; Curi \& Franzmeier, 1987; Busacca \& Singer, 1989; Esser et al., 1991; Plichta \& Kuczynska, 1991; Jeng \& Bergseth, 1992; Piccolo \& Celano, 1992; Angelone et al., 1993; Jing-Sheng et al., 1993; Pettry \& Switzer, 1993; Xing \& Dudas, 1993; Ferreira et al., 1994). Assim, a caracterização de diferentes classes de solo e a inter-relação com o material que lhe deu origem torna-se possível. Jing-Sheng et al. (1993) avaliaram as tendências geográficas dos conteúdos de metais pesados em solos chineses derivados de basalto, granito e siltito, identificando, nesta ordem, a seqüência decrescente de conteúdo dos metais pesados, com variações, conforme a localização geográfica, especificamente em relação ao basalto e o siltito, as quais revelaram-se similar às variações do conteúdo de óxidos férricos do solo. Resende et al. (1986) e Curi \& Franzmeier (1987) ao comentarem a respeito dessas tendências em solos brasileiros, corroboram os resultados obtidos por Jing-Sheng et al. (1993). Todavia, a avaliação e definição da intensidade de influência de um ou outro material de origem nos solos formados parece ser a maior dificuldade encontrada, principalmente quando se considera em uma mesma área mais de uma formação geológica, uma vez que há uma interposição das contribuições no material intemperizado formador do solo.

O objetivo deste trabalho foi determinar os teores de metais pesados em amostras de solos de uma topolitosequiência do Triângulo Mineiro, Estado de Minas Gerais, para testar a hipótese de que tais elementos podem ser utilizados na identificação e separação da influência do material de origem.

\section{MATERIAL E MÉTODOS}

A área de estudo da topolitoseqüência situa-se na região fisiográfica denominada Triângulo Mineiro, Minas Gerais, a qual pertence à bacia do rio Paranaíba, que por sua vez é componente da bacia hidrográfica do rio Paraná. Os solos foram coletados em pontos ao longo da rodovia BR-050, entre os municípios de Uberlândia e Araguari, no trecho compreendido entre o rio Araguari e a sede do primeiro município (Fig. 1) (Minas Gerais, 1976).

A seleção dos pontos de amostragem considerou as variações na paisagem que refletissem os diferentes tipos de materiais de origem (gnaisse-basaltoarenito+sedimentos) da área de estudo, principalmente no que se refere a mudanças no relevo, altitude, afloramentos de rochas, cor dos solos e textura. No total foram coletados 24 solos, procurando-se amostrar pontos em áreas representativas dos diferentes materiais litológicos e suas áreas de transição - estas com maior intensidade, ou seja, a intervalos de distância menores, de acordo com as observações de campo e a expectativa de amostragem detalhada.

Foram coletadas amostras de solos nas profundidades de 0 a $10 \mathrm{~cm}$ (1) e 50 a $60 \mathrm{~cm}$ (2), correspondentes aos horizontes A e B, aproveitando-se cortes ao longo do leito da estrada, ou com a abertura de uma pequena trincheira, considerando-se uma distância mínima de $50 \mathrm{~m}$ do leito da estrada.

Foi feita a descrição dos perfis, nas profundidades de coleta, conforme Lemos \& Santos (1984), à exceção do transecto. O posicionamento geográfico foi determinado com a utilização de equipamento de posicionamento geográfico global (GPS) e a altitude obtida com altímetro de mão.

As frações cascalho e calhaus foram determinadas por peneiramento, lavagem e posterior quantificação após secagem em estufa a $105^{\circ} \mathrm{C}$. O método da pipeta foi o utilizado para determinação da argila e silte, com dispersão efetuada quimicamente $(\mathrm{NaOH}, 0,1 \mathrm{~mol} / \mathrm{L}) \mathrm{e}$ mecanicamente (agitação rápida), e a areia grossa e fina, por tamizagem. Para a classificação textural, foi feito o uso do triângulo textural e dos valores obtidos na análise granulométrica (Embrapa, 1979). As cores dos solos foram determinadas por comparação com padrões contidos na Carta de Cores de Munsell (Munsell Color, 1975), em amostras de TFSA secas e ligeiramente umedecidas.

$\mathrm{O}$ pH em água e em $\mathrm{KCl} 1 \mathrm{~mol} / \mathrm{L}$ foram determinados potenciometricamente em suspensão, empregando-se relação 1:2,5 de solo:água e solo: $\mathrm{KCl}$, após uma hora, no mínimo, de repouso e agitação da suspensão antes da 
leitura, conforme Embrapa (1979). $\mathrm{Al}^{3+}, \mathrm{Ca}^{2+}$ e $\mathrm{Mg}^{2+}$ trocáveis foram extraídos com $\mathrm{KCl} 1 \mathrm{~mol} / \mathrm{L}$, na proporção 1:20, sendo $\mathrm{Al}^{3+}$ determinado por titulação com $\mathrm{NaOH}$

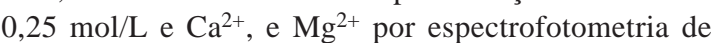
absorção atômica, segundo Defelipo \& Ribeiro (1981). Extraíram-se K e P disponíveis com solução $\mathrm{HCl} 0,05 \mathrm{~mol} / \mathrm{L} \mathrm{e}_{2} \mathrm{SO}_{4}$ 0,0125 mol/L (Mehlich-1), na proporção $1: 10$. O K foi determinado por fotometria de chama, e o P, por colorimetria na presença de ácido ascórbico, conforme Defelipo \& Ribeiro (1981). A acidez potencial $\left(\mathrm{H}^{+}+\mathrm{Al}^{3+}\right)$ foi determinada com $\mathrm{Ca}(\mathrm{OAc})_{2} 0,5 \mathrm{~mol} / \mathrm{L}$, ajustado para $\mathrm{pH} 7,0$, na proporção 1:15 e titulação com $\mathrm{NaOH}$ 0,0606 mol/L, segundo Embrapa (1979).

A determinação de $\mathrm{Fe}_{2} \mathrm{O}_{3}, \mathrm{Al}_{2} \mathrm{O}_{3}, \mathrm{SiO}_{2}, \mathrm{TiO}_{2}$ e $\mathrm{P}_{2} \mathrm{O}_{5}$ consistiu de pré-tratamento da argila para a extração de $\mathrm{Fe}$, $\mathrm{Al}$, Ti e $\mathrm{Si}$, após contato com $\mathrm{H}_{2} \mathrm{SO}_{4}$ 1:1 (volume), aquecendo até a fervura, sob refluxo, com posterior resfriamento, diluição e filtração. No resíduo determinou-se $\mathrm{SiO}_{2}$, e no

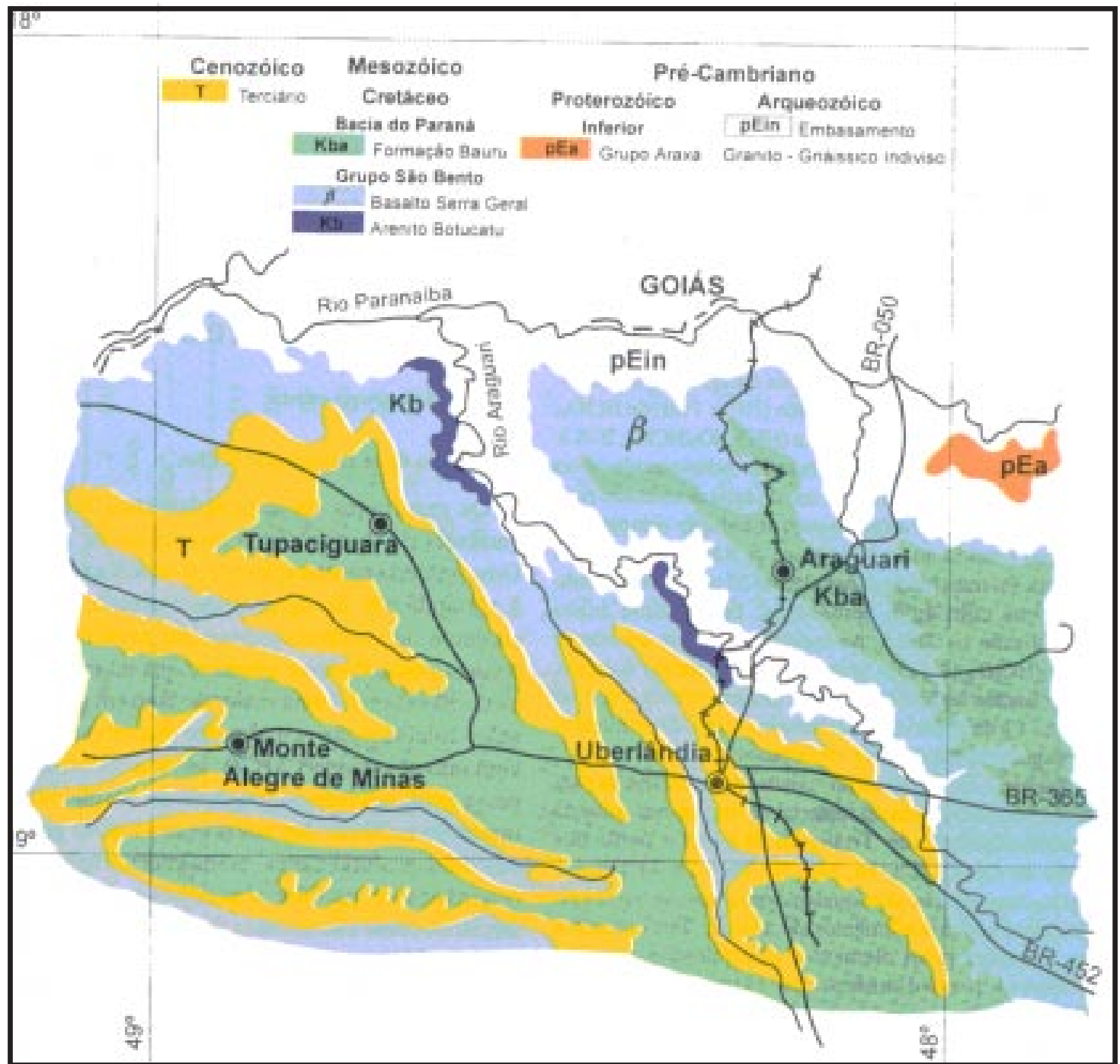

FIG. 1. Mapa geológico e de localização da topolitoseqüência na região do Triângulo Mineiro, Estado de Minas Gerais (Fonte: Minas Gerais, 1976). 
filtrado, $\mathrm{Fe}_{2} \mathrm{O}_{3}, \mathrm{Al}_{2} \mathrm{O}_{3}, \mathrm{TiO}_{2}$ e $\mathrm{P}_{2} \mathrm{O}_{5}$, conforme Embrapa (1979). As relações moleculares $\mathrm{Ki}, \mathrm{Kr}$ e $\mathrm{Al}_{2} \mathrm{O}_{3} / \mathrm{Fe}_{2} \mathrm{O}_{3}$ também foram calculadas.

Na Tabela 1 são apresentadas a identificação, características e propriedades químicas e físicas dos solos.

A determinação de $\mathrm{Cd}, \mathrm{Cr}, \mathrm{Cu}, \mathrm{Fe}, \mathrm{Mn}, \mathrm{Ni}, \mathrm{Pb}$ e $\mathrm{Zn}$ foi feita por dois diferentes processos de ataque ácido para obtenção dos teores totais. No primeiro, utilizaram-se $25 \mathrm{~mL}$ de ácidos nítrico $\left(\mathrm{HNO}_{3}\right)$ e perclórico $\left(\mathrm{HClO}_{4}\right)$ concentrados, na proporção 4:1 (volume), em erlemeyers de $125 \mathrm{~mL}$ e chapa quente, com aumento gradual da temperatura. Foram usados $2 \mathrm{~g}$ de TFSA, triturados em almofariz de ágata e tempo de contato prévio de 12 horas. Após secagem, adicionaram-se $3 \mathrm{~mL}$ de ácido clorídrico $(\mathrm{HCl})$ concentrado, transferindo-se para balão de $25 \mathrm{~mL}$ com água destilada e filtragem com papel-filtro quantitativo lento. Como não houve dissolução completa, o resíduo foi recolhido, secado e armazenado para posterior análise. Foram determinados os elementos por espectrofotometria de absorção atômica; realizaram-se 10 repetições.

No segundo procedimento, em beckers de teflon e em duplicatas, adicionaram-se $6 \mathrm{~mL}$ de $\mathrm{HNO}_{3}$ e $1 \mathrm{~mL}$ de $\mathrm{HClO}_{4}$ concentrados, com elevação gradual de temperatura em chapa quente e banho de areia. Após secagem, foram adicionados $10 \mathrm{~mL}$ de ácido fluorídrico (HF) e $1 \mathrm{~mL}$ de $\mathrm{HClO}_{4}$, aquecendo novamente até secura $\left(\mathrm{HF} / \mathrm{HNO}_{3} / \mathrm{HClO}_{4}, 20: 6: 3\right.$, volume). Este último passo foi repetido adicionando-se ao final $5 \mathrm{~mL}$ de HCl, $6 \mathrm{~mol} / \mathrm{L}$ e $5 \mathrm{~mL}$ de água deionizada, com aquecimento por 10 minutos para dissolução completa do material contido no becker (Mann \& Ritchie, 1993). Em seguida, foi transferido para balão de $25 \mathrm{~mL}$, completando o volume com água deionizada. Este ataque ácido foi realizado na TFSA, no resíduo originário do ataque $\mathrm{HNO}_{3} / \mathrm{HClO}_{4}$ (4:1) do primeiro procedimento e argila, sendo usados nos dois primeiros procedimentos $2 \mathrm{~g}$, e no último, $1 \mathrm{~g}$ de material. Os elementos foram determinados por espectrofotometria de absorção atômica.

Técnicas de análise multivariada foram utilizadas para o estudo da influência do material de origem nos solos da topolitoseqüência do Triângulo Mineiro a partir dos teores de $\mathrm{Cd}, \mathrm{Cr}, \mathrm{Cu}, \mathrm{Fe}, \mathrm{Mn}, \mathrm{Ni}, \mathrm{Pb}$ e $\mathrm{Zn}$ da TFSA e argila, formadores dos quatro conjuntos de variáveis utilizados nos procedimentos de análise. Tais conjuntos são definidos pelos elementos determinados nos dois primeiros procedimentos de extração total com $\mathrm{HNO}_{3} / \mathrm{HClO}_{4}(4: 1)$, concentrados, na TFSA (C1) e $\mathrm{HF} / \mathrm{HNO}_{3} / \mathrm{HClO}_{4}(20: 6: 3)$, concentrados, na TFSA (C2) e na argila (C3), e, por último, um conjunto de variáveis composto pelos conjuntos anteriores: $\mathrm{C} 4=\mathrm{C} 1+\mathrm{C} 2+\mathrm{C} 3$, organizados nessa ordem, respeitando-se a disposição individual das variáveis em cada grupo.
As técnicas utilizadas podem ser divididas em análises de agrupamento e discriminante. Para o agrupamento houve o envolvimento da distância generalizada de Mahalanobis (DGM), uma medida de dissimilaridade ( $\left.\mathrm{D}^{2}\right)$ aplicável nos casos em que existe correlação residual entre as características ou propriedades analisadas (Mahalanobis, 1936), como base para a aplicação do método de otimização de Tocher, citado por Rao (1952), e da análise por variáveis canônicas (Rao, 1952). A análise discriminante foi realizada, de acordo com Anderson (1958) com a finalidade de classificar solos de origem desconhecida em agrupamentos já conhecidos, ou seja, considerou-se a existência de solos de origem gnaíssica, basáltica e arenítica típicas, definidos em campo e com base em levantamentos geológicos da área.

Os fundamentos teóricos e exemplos de aplicação são apresentados por Cruz \& Regazzi (1994), e para execução dos procedimentos estatísticos foi utilizado o programa Análise de Modelos Biométricos Aplicados ao Melhoramento Genético - Programa Genes, desenvolvido na Universidade Federal de Viçosa (UFV).

\section{RESULTADOS E DISCUSSÃO}

\section{Análise de agrupamento}

\section{Método de otimização de Tocher}

Os resultados da aplicação desta técnica às variáveis em estudo são apresentadas na Tabela 2. Uma primeira constatação que se pode fazer refere-se à variabilidade do agrupamento obtido, tanto por conjunto de variáveis quanto por profundidade de coleta adotada. Pode-se observar que na profundidade de 50-60 cm há uma maior divisão em grupos dos solos coletados, principalmente quando se utiliza o conjunto de variáveis 3 (C3); ou seja, identificou-se nesta profundidade e conjunto de variáveis, uma maior diversidade dos solos coletados. O grupo 1, nas duas profundidades, normalmente engloba solos originários de gnaisse e arenito+sedimentos do terciário, como são os casos dos solos 1, 2, 3 e 4 e 21, 22, 23, 24, considerados, pelas observações de campo, como tipicamente formados a partir de gnaisse e arenito+sedimentos do terciário, respectivamente, e alguns outros que podem estar situados nas áreas de transição, ou não, com o basalto. $\mathrm{O}$ fato de se agrupar solos tão diferentes pode ser atribuído a uma questão numérica, ou seja, à semelhança de teores 
TABELA 1. Identificação, características e propriedades químicas e físicas dos solos, na profundidade de $50-60 \mathrm{~cm}$.

\begin{tabular}{|c|c|c|c|c|c|c|c|c|c|c|c|c|c|c|c|c|c|c|c|c|c|}
\hline \multicolumn{2}{|c|}{ Solos } & \multicolumn{2}{|c|}{$\mathrm{pH}(1: 2,5)$} & \multirow[t]{2}{*}{$\mathrm{Ca}^{2+}$} & \multirow[t]{2}{*}{$\mathrm{Mg}^{2+}$} & \multirow[t]{2}{*}{$\mathrm{Al}^{3+}$} & \multirow[t]{2}{*}{$\mathrm{H}^{+}+\mathrm{Al}^{3+}$} & \multirow{2}{*}{\multicolumn{2}{|c|}{$\begin{array}{cc}\mathrm{K}^{+} & \mathrm{P} \\
\text { disp. } & \text { disp. } \\
--\left(\mathrm{mg} / \mathrm{dm}^{3}\right)--\end{array}$}} & \multirow{2}{*}{$\mathrm{SiO}_{2}$} & \multirow[t]{2}{*}{$\mathrm{Al}_{2} \mathrm{O}_{3}$} & \multirow[t]{2}{*}{$\mathrm{Fe}_{2} \mathrm{O}_{3}$} & \multirow[t]{2}{*}{$\mathrm{TiO}_{2}$} & \multirow[t]{2}{*}{$\mathrm{P}_{2} \mathrm{O}_{5}$} & \multirow[t]{2}{*}{$\mathrm{Ki}$} & \multirow[t]{2}{*}{$\mathrm{Kr}$} & \multirow{2}{*}{$\begin{array}{l}\mathrm{Al}_{2} \mathrm{O}_{3} / \\
\mathrm{Fe}_{2} \mathrm{O}_{3}\end{array}$} & \multirow{2}{*}{$\begin{array}{l}\text { Areia } \\
\text { grossa }\end{array}$} & \multirow{2}{*}{$\begin{array}{c}\text { Areia } \\
\text { fina }\end{array}$} & \multirow[t]{2}{*}{ Silte } & \multirow[t]{2}{*}{ Argili } \\
\hline № & Classe $^{1}$ & Água & $\begin{array}{c}\mathrm{KCl} \\
\left(1 \mathrm{~mol} / \mathrm{dm}^{3}\right)\end{array}$ & & & & & & & & & & & & & & & & & & \\
\hline 1 & $\mathrm{C} / \mathrm{L}$ & 5,4 & 4,6 & 1,6 & 0,5 & 0,3 & 3,9 & 59 & 1,3 & 273 & 249 & 115 & 13,2 & 2,1 & 1,86 & 1,44 & 3,40 & 230 & 260 & 130 & 380 \\
\hline 2 & PV & 5,4 & 4,7 & 1,1 & 0,5 & 0,0 & 2,7 & 62 & 0,8 & 273 & 262 & 104 & 10,0 & 8,0 & 1,77 & 1,41 & 3,95 & 280 & 210 & 70 & 440 \\
\hline 3 & PV & 4,9 & 4,2 & 0,6 & 0,8 & 0,4 & 3,9 & 80 & 1,5 & 313 & 246 & 91 & 9,0 & 8,0 & 2,16 & 1,75 & 4,24 & 220 & 220 & 150 & 410 \\
\hline 4 & $\mathrm{C}$ & 5,2 & 4,2 & 1,2 & 0,4 & 0,0 & 3,6 & 85 & 1,1 & 300 & 254 & 96 & 9,4 & 1,1 & 2,01 & 1,62 & 4,15 & 310 & 320 & 110 & 260 \\
\hline 5 & PE & 4,9 & 4,3 & 0,6 & 0,8 & 0,0 & 1,5 & 163 & 0,2 & 243 & 283 & 151 & 15,4 & 1,3 & 1,46 & 1,09 & 2,94 & 140 & 200 & 200 & 460 \\
\hline 6 & $\mathrm{PE} / \mathrm{TE}$ & 5,5 & 4,8 & 1,9 & 0,9 & 0,0 & 3,6 & 31 & 0,2 & 233 & 238 & 190 & 17,4 & 2,6 & 1,66 & 1,10 & 1,97 & 210 & 140 & 170 & 480 \\
\hline 7 & $\mathrm{~L}$ & 5,4 & 4,5 & 2,6 & 3,1 & 12,9 & 16,5 & 25 & 7,7 & 334 & 190 & 139 & 9,6 & 2,7 & 2,99 & 2,03 & 2,15 & 210 & 310 & 250 & 230 \\
\hline 8 & LR/TE & 6,0 & 5,1 & 4,4 & 2,5 & 0,0 & 3,6 & 397 & 0,5 & 239 & 214 & 174 & 16,2 & 1,7 & 1,90 & 1,25 & 1,93 & 30 & 100 & 250 & 620 \\
\hline 9 & LR & 5,3 & 4,2 & 1,1 & 0,4 & 0,7 & 5,4 & 28 & 0,2 & 222 & 247 & 190 & 15,6 & 1,6 & 1,53 & 1,02 & 2,04 & 30 & 160 & 200 & 610 \\
\hline 10 & LR & 5,0 & 4,4 & 0,8 & 0,2 & 0,1 & 7,5 & 19 & 0,6 & 185 & 235 & 239 & 35,6 & 2,1 & 1,34 & 0,81 & 1,54 & 50 & 60 & 220 & 670 \\
\hline 11 & $\mathrm{~L}$ & 5,3 & 4,2 & 1,2 & 0,7 & 0,9 & 9,6 & 37 & 0,8 & 237 & 202 & 224 & 32,8 & 2,9 & 1,99 & 1,17 & 1,41 & 40 & 40 & 320 & 600 \\
\hline 12 & LE & 5,3 & 4,8 & 1,2 & 0,2 & 0,0 & 7,8 & 13 & 0,2 & 123 & 234 & 258 & 36,4 & 2,6 & 0,89 & 0,52 & 1,42 & 140 & 120 & 290 & 450 \\
\hline 13 & LR & 5,6 & 5,0 & 1,3 & 0,2 & 0,0 & 7,2 & 10 & 0,2 & 92 & 245 & 258 & 36,0 & 2,9 & 0,64 & 0,38 & 1,49 & 140 & 100 & 290 & 470 \\
\hline 14 & LR & 5,1 & 4,5 & 1,0 & 0,0 & 0,0 & 6,9 & 15 & 0,2 & 84 & 268 & 263 & 39,2 & 2,2 & 0,53 & 0,33 & 1,60 & 120 & 100 & 260 & 520 \\
\hline 15 & LR & 4,9 & 4,3 & 0,8 & 0,0 & 0,0 & 2,4 & 7 & 0,5 & 35 & 248 & 311 & 40,0 & 2,2 & 0,24 & 0,13 & 1,25 & 180 & 110 & 310 & 400 \\
\hline 16 & LR & 4,9 & 4,3 & 0,8 & 0,0 & 0,0 & 2,4 & 7 & 0,5 & 35 & 248 & 311 & 40,0 & 2,2 & 0,24 & 0,13 & 1,25 & 170 & 130 & 260 & 440 \\
\hline 17 & LE & 5,4 & 4,6 & 1,4 & 0,7 & 1,3 & 5,4 & 51 & 0,2 & 242 & 235 & 186 & 18,4 & 1,7 & 1,75 & 1,16 & 1,98 & 70 & 80 & 260 & 590 \\
\hline 18 & LR & 5,0 & 4,3 & 0,7 & 0,1 & 0,0 & 2,7 & 14 & 0,2 & 164 & 253 & 206 & 28,0 & 2,5 & 1,10 & 0,72 & 1,93 & 130 & 220 & 150 & 500 \\
\hline 19 & LR & 4,8 & 4,1 & 0,4 & 0,0 & 0,0 & 2,4 & 7 & 0,3 & 154 & 257 & 215 & 35,2 & 2,5 & 1,02 & 0,66 & 1,88 & 250 & 280 & 70 & 400 \\
\hline 20 & LE & 4,8 & 4,2 & 0,1 & 0,1 & 0,0 & 1,8 & 5 & 1,8 & 152 & 261 & 167 & 22,4 & 1,2 & 0,99 & 0,70 & 2,45 & 290 & 290 & 90 & 330 \\
\hline 21 & LR/LE & 5,3 & 4,6 & 1,1 & 0,3 & 0,0 & 3,0 & 10 & 0,2 & 148 & 230 & 224 & 50,8 & 2,6 & 1,09 & 0,67 & 1,61 & 260 & 240 & 80 & 420 \\
\hline 22 & LE & 4,7 & 4,0 & 0,1 & 0,0 & 0,0 & 1,8 & 9 & 0,2 & 165 & 279 & 142 & 23,2 & 1,0 & 1,00 & 0,76 & 3,08 & 330 & 220 & 50 & 400 \\
\hline 23 & LE & 4,9 & 4,3 & 0,4 & 0,1 & 0,0 & 2,7 & 11 & 0,2 & 202 & 283 & 136 & 16,4 & 1,0 & 1,21 & 0,93 & 3,27 & 320 & 160 & 50 & 470 \\
\hline 24 & LE & 4,7 & 4,0 & 0,3 & 0,0 & 0,1 & 3,6 & 10 & 0,2 & 187 & 304 & 126 & 19,8 & 0,9 & 1,04 & 0,83 & 3,79 & 150 & 70 & 90 & 690 \\
\hline
\end{tabular}


totais dos solos coletados, obtidos pelos métodos de extração usados. Porém, não inviabiliza a separação qualitativa desses solos quanto à sua origem, pois, na seqüência coletada, eles se encontram separados pelos solos originários de basalto, definindo mais especificamente a origem destes. Entretanto, os solos originários de basalto foram agrupados algumas vezes em um único grupo, e em outros, em até três, nos casos dos solos 11, 12, 13 e 14 , fato que se repete nas demais técnicas multivariadas utilizadas, constatando que a similaridade ou diversidade desses solos foi função das características das variáveis, quanto a fração analisada, profundidade de coleta e organização destas.

TABELA 2. Agrupamento dos 24 solos coletados na topolitoseqüência do Triângulo Mineiro, pelo método de otimização de Tocher com base na distância generalizada de Mahalanobis, utilizando-se conjuntos de variáveis (C1, C2, C3 e C4), nas profundidades de 0-10 cm e 50-60 cm.

\begin{tabular}{|c|c|c|c|c|c|}
\hline \multirow{2}{*}{$\begin{array}{l}\text { Profundidade } \\
\quad(\mathrm{cm})\end{array}$} & \multirow[t]{2}{*}{ Grupo } & \multicolumn{4}{|c|}{ Conjunto de variáveis ${ }^{1}$} \\
\hline & & $\mathrm{C} 1$ & $\mathrm{C} 2$ & $\mathrm{C} 3$ & $\mathrm{C} 4$ \\
\hline \multirow[t]{6}{*}{$0-10$} & 1 & $\begin{array}{l}22,23,20,24,19,21, \\
18,5,4,2,3,6,9,17, \\
16,15,10,1,8\end{array}$ & $\begin{array}{l}2,4,23,22,20,24,3 \\
5,21,19,1,6,18,7 \\
9,17,16,15\end{array}$ & $\begin{array}{l}23,24,22,20,6,5,2,19 \\
7,8,18,3,21,4,9,10\end{array}$ & $\begin{array}{l}2,3,23,4,22,21,24, \\
5,6,18,8\end{array}$ \\
\hline & 2 & 13,14 & $10,11,8$ & $15,16,17,11,14$ & 19,20 \\
\hline & 3 & 12 & 13,14 & 12,13 & $15,17,16,11,10$ \\
\hline & 4 & 11 & 12 & 1 & $12,14,13$ \\
\hline & 5 & 7 & & & 1,7 \\
\hline & 6 & & & & 9 \\
\hline \multirow[t]{9}{*}{$50-60$} & 1 & $\begin{array}{l}20,23,22,24,19,21 \\
4,5,2,1,3,18,6,8 \\
9,17,15,16\end{array}$ & $\begin{array}{l}1,3,2,5,4,23,22 \\
21,20,24,19,8\end{array}$ & $\begin{array}{l}20,24,23,22,2,5,19, \\
21,6,4\end{array}$ & $\begin{array}{l}20,23,22,4,24,3,2,1, \\
21,19,5,8,6\end{array}$ \\
\hline & 2 & $10,11,7$ & $\begin{array}{l}10,17,11,9,18,7 \\
16\end{array}$ & $7,8,9$ & $\begin{array}{l}7,9,17,15,16,10,18 \\
14,11\end{array}$ \\
\hline & 3 & 13,14 & 13,14 & $16,17,14$ & 12,13 \\
\hline & 4 & 12 & 12 & 10,11 & \\
\hline & 5 & & 15 & 12,13 & \\
\hline & 6 & & 6 & 18 & \\
\hline & 7 & & & 15 & \\
\hline & 8 & & & 1 & \\
\hline & 9 & & & 3 & \\
\hline
\end{tabular}

${ }^{1} \mathrm{C} 1, \mathrm{C} 2, \mathrm{C} 3$ e C4 são conjuntos de variáveis formados pelos teores de $\mathrm{Cd}, \mathrm{Cr}, \mathrm{Cu}, \mathrm{Fe}, \mathrm{Mn}, \mathrm{Ni}, \mathrm{Pb}$ e Zn, em mg/kg, extraídos pelos procedimentos de extração total com $\mathrm{HNO}_{3} / \mathrm{HClO}_{4}(4: 1)(\mathrm{C} 1)$ e $\mathrm{HF} / \mathrm{HNO}_{3} / \mathrm{HClO}_{4}(20: 6: 3)(\mathrm{C} 2)$ na TFSA e $\mathrm{HF} / \mathrm{HNO}_{3} / \mathrm{HClO}_{4}$ (20:6:3) na argila (C3); o C4 é formado pelos três primeiros conjuntos, ou seja, os elementos extraídos com $\mathrm{HNO}_{3} / \mathrm{HClO}_{4}(4: 1)$ e $\mathrm{HF} / \mathrm{HNO}_{3} / \mathrm{HClO}_{4}(20: 6: 3)$ na TFSA e argila, respeitando-se a disposição individual das variáveis em cada grupo: $\mathrm{C} 4=\mathrm{C} 1+\mathrm{C} 2+\mathrm{C} 3$. 
Além desses aspectos, deve-se ressaltar, também, que observações de campo indicaram variações em relação à própria composição do basalto ou mesmo a processos de derramamento ocorridos em diferentes épocas, possibilitando, assim, a alternância com outros tipos de materiais. Jing-Sheng et al. (1993) também constataram variações dos teores de metais pesados em vários solos com origem basáltica no leste da China.

De modo geral, o método de agrupamento de Tocher, com base na distância generalizada de Mahalanobis, permite classificar os solos 5 e 6 e 19 e 20 como formados a partir do gnaisse e arenito+sedimentos do terciário, visto que estes se repetem em todos os grupos que também constam os solos considerados típicos dessas áreas, com exceção do 6, que não foi classificado como tal no conjunto $\mathrm{C} 2$ e profundidade 2 . Como esses dois materiais de origem situam-se entre o basalto, a própria distância entre eles sugere a classificação proposta. Já os demais solos, considerados como de localização em áreas de transição, são, algumas vezes, agrupados como originários de basalto, ou então, gnaisse e arenito+sedimentos do terciário, alternadamente. Com as demais técnicas utilizadas tentar-se-á definir de forma mais precisa qual a origem desses solos.

Com relação às variáveis utilizadas neste estudo, elas foram definidas em conjuntos, formados a partir do tipo de extração, fração utilizada e profundidade de estudo. A princípio bastaria um único tipo de extração total. Contudo, as razões para a adoção de tal procedimento estão em função, principalmente, da identificação das influências que as variáveis teriam sobre a classificação obtida. É certo que existiram diferenças quanto aos teores em um e outro solo, bem como forma de extração, mas com tendências gerais semelhantes. Porém, tais diferenças poderão ser de grande auxílio na definição posterior da origem dos demais solos coletados, uma vez que as pequenas diferenças observadas levaram ao agrupamento diferenciado dos solos. Determinações em diferentes profundidades permitem uma visão mais global da identificação a ser feita, uma vez que o maior ou menor número de grupos formados podem ser indicativos da proximidade de solos de áreas de transição em um grupo preestabelecido e conhecido ou não, como é o caso do presente. A definição conjunta com informações obtidas por outros meios será facilitada com adoção desta prática. Já com relação aos conjuntos de variáveis, observa-se que há uma diferenciação acentuada dos agrupamentos obtidos, o que dificulta uma definição mais clara da melhor recomendação.

\section{Variáveis canônicas}

Em sete dos oito conjuntos de variáveis e profundidades analisados, observou-se que as duas primeiras variáveis canônicas contribuíram efetivamente para explicar a dispersão dos 24 solos coletados, viabilizando, portanto, a utilização dessa técnica (Tabela 3). Deve-se destacar os conjuntos C1 e C4, o primeiro formado com as oito variáveis estudadas e dez repetições e o segundo composto de 24 variáveis, originário da junção dos três primeiros conjuntos, mas somente com duas repetições, nos quais as contribuições das duas primeiras variáveis canônicas na profundidade 1 foram inferiores a $80 \%$ e superiores a $99 \%$, respectivamente. Tal fato pode ser indicativo de que um número maior de variáveis é mais explicativo quanto a divergência existente, tornando mais precisos os processos de avaliação da similaridade entre os solos estudados, dispensando o cuidado extremo com o número de repetições, ou mesmo, um indicativo da menor eficiência de extração da mistura $\mathrm{HNO}_{3} / \mathrm{HClO}_{4}$, na proporção utilizada (4:1), uma vez que os teores observados apresentam-se relativamente menores que os obtidos com a mistura de $\mathrm{HF} / \mathrm{HNO}_{3} / \mathrm{HClO}_{4}(20: 6: 3)$.

Observou-se certa concordância entre os resultados obtidos pelo método de agrupamento de Tocher (Tabela 2) e a técnica de variáveis canônicas (Tabela 3 e Figs. 2 e 3), principalmente quanto aos solos originários de gnaisse e arenito+sedimentos do terciário, o que de certa forma deveria ser esperado, já que se trata de uma técnica de avaliação de similaridade ou de dissimilaridade entre indivíduos. Já com relação aos solos originários de basalto, constatou-se a formação de um grupo mais disperso, como é o caso do solos 12, 13 e 14, e, à parte, o 11, este certamente por apresentar teores diferenciados dos demais em função da maior proximidade com o material de origem, já que se trata de um Litossolo, enquanto os demais caracteri- 
zam-se como Latossolos (Tabela 1), além do aspecto comentado anteriormente, quanto à composição do basalto. Para o enquadramento dos demais solos quanto à influência de um material de origem ou outro, adotou-se a estratégia de avaliar as distâncias relativas destes, com relação aos grupos bem definidos, como constatado para os de gnaisse e arenito+sedimentos do terciário, ou individualmente, por intermédio de solos típicos de um ou outro material, como os de basalto, conforme definição em campo, durante a coleta. Diante disso, serão apresentados somente os resultados relativos ao grupo $\mathrm{C} 4$, uma vez que foi o conjunto de variáveis canônicas que efetivamente explicou a dispersão gráfica, porém, com comentários acerca dos demais grupos de variáveis estudados.

Nos solos originários de gnaisse e arenito+sedimentos do terciário, agrupados quase em um único grupo pelo agrupamento de Tocher (Tabelas 2 e 4), o conjunto $C 1$, quando analisado na profundidade 1 , não apresentou dispersão que permita uma definição clara dos grupos, o que pode estar relacionado ao fato de que as duas primeiras variáveis canônicas não envolveram $80 \%$ da variância acumulada, no caso $77 \%$ (Tabela 3). Como haveria necessidade de dispersão tridimensional para tão pouca diferença, o mesmo não foi feito, o que certamente não levaria a uma melhora sensível do quadro apresentado. Todavia, pôde-se observar uma tendência clara de isolamento dos solos com forte influência do basalto: 11, 12 e 13, com destaque para o 7 e 14 . Os solos 10,15 e 16, por sua vez encontramse mais próximos do grupo com influência de gnaisse e arenito+sedimentos do terciário. Já quando avaliou-se na profundidade 2, constatou-se comportamento semelhante ao observado para os solos de

TABELA 3. Estimativa dos autovalores $\left(\lambda_{\mathrm{i}}\right)$ e da porcentagem de contribuição $\left(\% \lambda_{\mathrm{i}}\right)$ obtidos da análise de variáveis canônicas, aplicada aos 24 solos coletados na topolitoseqüência do Triângulo Mineiro, utilizando-se conjuntos de variáveis $(\mathrm{C} 1, \mathrm{C} 2, \mathrm{C} 3$ e $\mathrm{C4})$ nas profundidades de $0-10 \mathrm{~cm}$ e $50-60 \mathrm{~cm}$.

\begin{tabular}{|c|c|c|c|c|c|c|c|c|c|}
\hline \multirow{3}{*}{$\begin{array}{l}\text { Profundidade } \\
\quad(\mathrm{cm})\end{array}$} & \multirow{3}{*}{$\begin{array}{l}\text { Variável } \\
\text { canônica }\end{array}$} & \multicolumn{8}{|c|}{ Conjunto de variáveis ${ }^{1}$} \\
\hline & & \multicolumn{2}{|c|}{$\mathrm{C} 1$} & \multicolumn{2}{|c|}{$\mathrm{C} 2$} & \multicolumn{2}{|c|}{$\mathrm{C} 3$} & \multicolumn{2}{|c|}{$\mathrm{C} 4$} \\
\hline & & $\lambda_{\mathrm{i}}$ & $\% \lambda_{i}^{2}$ & $\lambda_{\mathrm{i}}$ & $\% \lambda_{\mathrm{i}}$ & $\lambda_{\mathrm{i}}$ & $\% \lambda_{\mathrm{i}}$ & $\lambda_{\mathrm{i}}$ & $\% \lambda_{\mathrm{i}}$ \\
\hline \multirow[t]{5}{*}{$0-10$} & 1 & 136,31 & 41,55 & $1.299,71$ & 72,69 & $2.254,08$ & 79,47 & $32.993 .182,00$ & 99,87 \\
\hline & 2 & 119,08 & 77,85 & 291,91 & 89,01 & 221,09 & 87,26 & $36.123,14$ & 99,98 \\
\hline & 3 & 42,05 & 90,67 & 87,99 & 93,94 & 188,50 & 93,91 & $3.927,65$ & 99,99 \\
\hline & 4 & 16,50 & 95,70 & 53,62 & 96,93 & 114,27 & 97,93 & $2.159,95$ & 99,99 \\
\hline & 5 & 9,29 & 98,53 & 31,01 & 98,67 & 44,72 & 99,51 & $1.029,45$ & 100,00 \\
\hline \multirow[t]{5}{*}{$50-60$} & 1 & 114,65 & 54,79 & $3.504,33$ & 80,02 & $1.361,60$ & 72,62 & $825.911,94$ & 97,12 \\
\hline & 2 & 54,03 & 80,61 & 567,44 & 92,98 & 222,28 & 84,48 & $19.643,45$ & 99,12 \\
\hline & 3 & 25,39 & 92,74 & 160,95 & 96,65 & 164,43 & 93,25 & $2.302,49$ & 99,70 \\
\hline & 4 & 5,89 & 95,55 & 73,30 & 98,33 & 44,69 & 95,63 & $1.220,73$ & 99,85 \\
\hline & 5 & 5,39 & 98,13 & 48,18 & 99,43 & 34,61 & 97,48 & 499,72 & 99,91 \\
\hline
\end{tabular}

1 C1, C2, C3 e C4 são conjuntos de variáveis formados pelos teores de $\mathrm{Cd}, \mathrm{Cr}, \mathrm{Cu}, \mathrm{Fe}, \mathrm{Mn}, \mathrm{Ni}, \mathrm{Pb}$ e Zn, em mg/kg, extraídos pelos procedimentos de extração total com $\mathrm{HNO}_{3} / \mathrm{HClO}_{4}(4: 1)(\mathrm{C} 1)$ e $\mathrm{HF} / \mathrm{HNO}_{3} / \mathrm{HClO}_{4}(20: 6: 3)(\mathrm{C} 2)$ na TFSA e $\mathrm{HF} / \mathrm{HNO}_{3} / \mathrm{HClO}_{4}$ (20:6:3) na argila (C3); o C4 é formado pelos três primeiros conjuntos, ou seja, os elementos extraídos com $\mathrm{HNO}_{3} / \mathrm{HClO}_{4}(4: 1)$ e $\mathrm{HF} / \mathrm{HNO}_{3} / \mathrm{HClO}_{4}(20: 6: 3)$ na TFSA e argila, respeitando-se a disposição individual das variáveis em cada grupo: $\mathrm{C} 4=\mathrm{C} 1+\mathrm{C} 2+\mathrm{C} 3$.

2 Valores em porcentagem acumulada. 
basalto e os destaques: 7 e 14, mas com relação aos solos agrupados comode gnaiss e e arenito+sedimentos do terciário, observou-se a exclusão de alguns com forte influência destes, tais como o 6, 8, 9 e 17, o que pode caracterizar um grupo intermediário entre esses solos. Os resultados obtidos, se comparados ao agrupamento proposto pelo método Tocher (Tabela 2), levam à indicação de pouca concordância, a um nível de exigência mais alto, pois o mesmo define bem os dois grupos, o que já não acontece quando se usam variáveis canônicas.

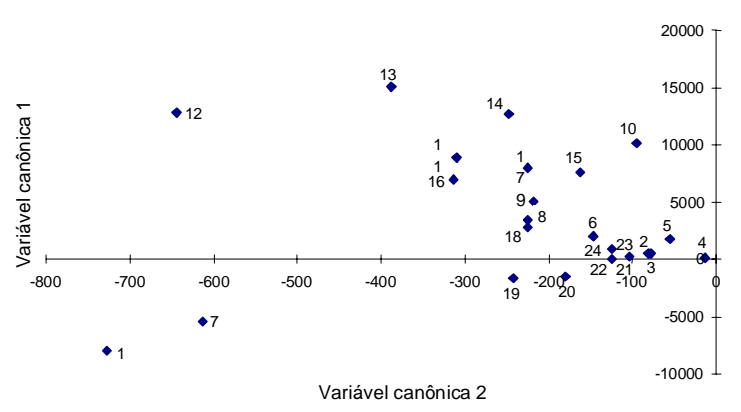

FIG. 2. Dispersão dos 24 solos coletados na topolitoseqüência do Triângulo Mineiro, utilizando-se conjunto de variáveis obtidas por extração com $\mathrm{HNO}_{3} / \mathrm{HClO}_{4}(4: 1)$ e HF/HNO $/ \mathrm{HClO}_{4}(20: 6: 3)$ (C4) na TFSA e argila, respectivamente, e profundidade de $0-10 \mathrm{~cm}$.

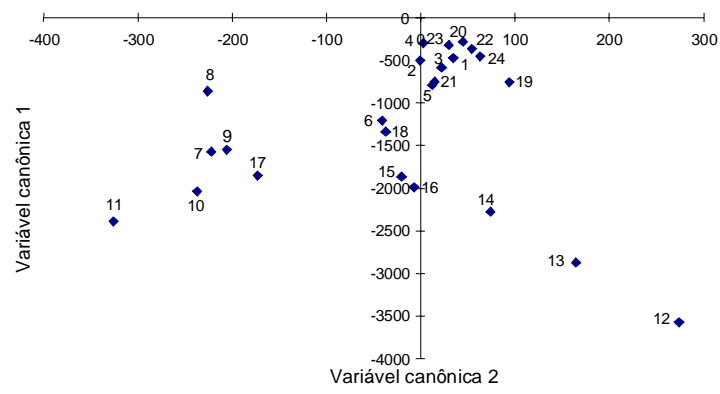

FIG.3. Dispersão dos 24 solos coletados na topolitosequiência do Triângulo Mineiro, utilizando-se conjunto de variáveis obtidas por extração com $\mathrm{HNO}_{3} / \mathrm{HClO}_{4} \quad(4: 1)$ e $\mathrm{HF} / \mathrm{HNO}_{3} / \mathrm{HClO}_{4}$ (20:6:3) (C4) na TFSA e argila, respectivamente, e profundidade de $50-60 \mathrm{~cm}$.
No conjunto $\mathrm{C} 2$, em que as duas primeiras variáveis canônicas contribuem com mais de $70 \%$ como forma explicativa da variabilidade (Tabela 3 ), confirma-se a tendência anterior quanto aos solos de gnaisse e arenito+sedimentos do terciário, observando-se um agrupamento muito mais definido, composto pelos solos de 1 a 5 e 19 a 24 , respectivamente, e, bastante próximos, os solos 6 e 18. A dispersão dos solos de basalto se repete, mas com maior proximidade de solos anteriormente indicados como de maior influência de gnaisse e arenito+sedimentos do terciário, como o 8, 9, 10, 15, 16 e 17, destacando-se, novamente o 7 e 14, como ocorrido com o C1. O mesmo pode ser observado na profundidade 2, utilizando-se o mesmo conjunto de variáveis.

Os grupos de solos originários de gnaisse e arenito+sedimentos do terciário apresentaram-se também bem definidos quando se utilizou o grupo $\mathrm{C} 3$, tanto na profundidade 1 quanto 2 , quando os teores de metais pesados foram determinados por $\mathrm{HF} / \mathrm{HNO}_{3} / \mathrm{HClO}_{4}(20: 6: 3)$ na fração argila, estando situado em posição intermediária aos conjuntos $\mathrm{C} 1 \mathrm{e}$ C2 quanto a contribuição das duas primeiras variáveis canônicas na variância explicada por estas (Tabela 3). Verificou-se que o solo 1 apresenta dispersão bastante diferenciada do observado, o que certamente deve estar associado às influências relativas à movimentação do lençol freático e material carreado a montante. Somente neste conjunto pôde-se observar esse comportamento e ocorrências, o que leva a crer que tais alterações estejam mais associadas à fração argila e não às mais grosseiras e, além de tudo, em superfície, uma vez que tal comportamento não se repete quando se avalia a profundidade de 50-60 cm (Figs. 2 e 3).

Com relação aos solos indicados como próximos no conjunto anterior aos agrupados como de gnaisse e arenito+sedimentos do terciário, 6 e 18, estes, no C3, podem ser considerados como inclusos neste agrupamento, tanto na profundidade 1 quanto na 2 , enquanto os demais continuam sendo considerados dispersos de forma próxima a solos individuais de basalto, à semelhança do conjunto $\mathrm{C} 2$, com exceção do 8 , neste caso, com dispersão que o caracteriza como pertencente ao grupo dos solos de gnaisse e arenito+sedimentos do terciário, nas duas profundi- 
dades estudadas. Comportamento contrário ao observado é o do 7, que neste conjunto está praticamente incluído no grupamento do gnaisse e arenito+sedimentos do terciário.

No conjunto $\mathrm{C} 4$, as duas primeiras variáveis canônicas explicaram mais de $99 \%$ da variância, o que indica que a dispersão dos diferentes solos estudados reflete de forma mais confiável a diversidade existente (Tabela 3), podendo ser indicativo de que um maior número de variáveis é preferível nesse tipo de estudo. Avaliando-se as Figs. 2 e 3, pode-se constatar que o comportamento observado com os demais conjuntos de variáveis, mesmo nos casos dos solos 1 e 8 e o grupo, até aqui considerado como próximo dos solos individuais de origem do basalto, 9, 10, 11,1516 e 17, se repete. Porém, o 7 diferencia-se do observado, apresentando-se disperso, como os solos de basalto, fato que se repete em outros conjuntos (C1 e C2) e profundidades estudados.

\section{Análise discriminante}

Os estudos de diversidade por meio das técnicas de agrupamento são complementados com a utilização de análise discriminante, visando avaliar a adequação da partição dos elementos agrupados proposta pelos métodos anteriores (Cruz \& Regazzi, 1994), tornando mais evidente, assim, a classificação dos diferentes solos estudados. Com tal objetivo, utilizou-se a técnica proposta por Anderson (1958), em que pressupondo uma otimização da classificação, quando se considera, simultaneamente, um conjunto de variáveis tomado em cada solo coletado, foram estabelecidas funções discriminantes a partir do conhecimento prévio dos elementos que pertencem a cada um dos materiais de origem estudados, utilizando os conjuntos de variáveis $\mathrm{C} 1, \mathrm{C} 2, \mathrm{C} 3$ e C4. Foram definidos os solos 2, 3 e 4; 13, 14 e 15 e 22, 23 e 24, como tipicamente formados a partir do gnaisse, basalto e arenito+sedimentos do terciário, respectivamente, de acordo com as observações de campo (Tabela 4).

As funções discriminantes dos três materiais de origem, considerando os solos citados, por conjunto de variáveis e profundidade estudados são uma combinação linear de alguns dos elementos estudados, sendo os diferentes solos classificados como de origem gnaíssica, basáltica ou arenítica, conforme o maior valor obtido pela substituição dos teores dos diferentes elementos analisados e que compõe as funções.

Entretanto, na obtenção das funções discriminantes, algumas variáveis foram eliminadas por haver uma dependência linear entre variáveis, proporcionando uma matriz de covariância singular. Como o processo de obtenção das funções discriminantes depende da inversa da matriz de covariância, a não-singularidade é indispensável e, portanto, a eliminação de algumas variáveis foi a alternativa que permitiu contornar o problema matemático exposto.

A classificação dos solos coletados quanto a sua origem é apresentada pela Tabela 4, em relação aos quatro conjuntos de variáveis e profundidades analisados. Essa classificação foi conduzida no âmbito da repetição, sendo apresentado, porém, a resultante que predominou em todas as repetições, e, nos casos em que uma única repetição ou mais proporcionou um enquadramento diferenciado de mais de um solo, foi apresentada em seqüência e, entre parênteses, o número de repetições em que isto foi constatado. A classificação geral, coincidente nas várias situações testadas, é também apresentada, como proposta final de classificação do solo analisado.

Da mesma forma, como observado nas outras técnicas testadas, não se constatou concordância entre as classificações obtidas, com variações entre conjuntos de variáveis e profundidades, porém, pode-se observar que essas diferenças concentram-se naquelas áreas de transição entre materiais de origem, como é o caso dos solos 8, 9, 16, 17 e 18, por exemplo. Mas mesmo assim pode-se propor a classificação geral na Tabela 4, tomando como referência o número de vezes em que há coincidência da classificação proposta para determinado solo, nas profundidades e conjuntos de variáveis estudadas, a partir dos resultados obtidos pela técnica da função discriminante de Anderson, como também os obtidos pelos métodos de agrupamento utilizados: Tocher e variáveis canônicas, com base na distância generalizada de Mahalanobis. Os solos de 1 a 9 podem ser classificados como formados a partir do 
gnaisse, mesmo quando, em alguns casos, o 6, 7, 8 e 9 ainda sejam classificados como de basalto, pois esta sugestão é suportada pelo número de vezes em que esses solos estão agrupados, tanto pelo método Tocher como pela dispersão obtida com a técnica das variáveis canônicas, estando próximos do grupo formado pelo gnaisse e arenito+sedimentos do terciário ou de solos específicos com esta origem.

A influência do basalto se faz sentir a partir do solo 10, mesmo quando este é ainda classificado como originário de gnaisse, pois se observa uma tendência geral para maior proximidade com os solos de origem evidente do basalto, tanto no agrupamento pelo método de Tocher quanto pela técnica de variáveis canônicas, à semelhança das observações feitas para definição da origem dos solos de gnaisse já comentados. Todas essas avaliações indicam que se pode atribuir a maior influência do basalto a partir do ponto de coleta do solo 10 até o 18; este último um tanto contraditório, em função de que nas demais técnicas utilizadas existem indicações de influência do arenito+sedimentos do terciário e numericamente de gnaisse, junto com o

TABELA4. Classificação individual e geral dos 24 solos coletados na topolitoseqüência do Triângulo Mineiro por conjunto de variáveis $(\mathrm{C} 1, \mathrm{C} 2, \mathrm{C} 3$ e $\mathrm{C} 4)$ e profundidades de $0-10 \mathrm{~cm}$ e $50-60 \mathrm{~cm}{ }^{1}$.

\begin{tabular}{|c|c|c|c|c|c|c|c|c|c|}
\hline \multirow[t]{3}{*}{ Solo } & \multicolumn{8}{|c|}{ Conjunto de variáveis ${ }^{2}$} & \multirow[t]{3}{*}{ Geral } \\
\hline & \multicolumn{2}{|c|}{$\mathrm{C} 1$} & \multicolumn{2}{|c|}{$\mathrm{C} 2$} & \multicolumn{2}{|c|}{$\mathrm{C} 3$} & \multicolumn{2}{|c|}{$\mathrm{C} 4$} & \\
\hline & $0-10$ & $50-60$ & $0-10$ & $50-60$ & $0-10$ & $50-60$ & $0-10$ & $50-60$ & \\
\hline 1 & $\mathrm{G}$ & $\bar{G}$ & $\bar{G}$ & $\bar{G}$ & $\bar{G}$ & $\mathrm{G}$ & $\mathrm{G}$ & $\mathrm{G}$ & $\bar{G}$ \\
\hline 2 & G & G & G & $\mathrm{G}$ & $\mathrm{G}$ & $\mathrm{G}$ & $\mathrm{G}$ & $\mathrm{G}$ & $\mathrm{G}$ \\
\hline 3 & G & G & G & G & G & G & G & G & G \\
\hline 4 & $\mathrm{G}$ & $\mathrm{G}$ & $\mathrm{G}$ & G & G & G & G & G & G \\
\hline 5 & $\mathrm{G}$ & G & G & G & G & G & $\mathrm{G}$ & G & G \\
\hline 6 & $\mathrm{G}$ & G & G & $\mathrm{G}$ & $\mathrm{G}$ & B & $\mathrm{G}$ & G & $\mathrm{G}$ \\
\hline 7 & $\mathrm{G}$ & G & G & B & $\mathrm{G}$ & B & $\mathrm{G}$ & G & $\mathrm{G}$ \\
\hline 8 & G & G & G & $\mathrm{G}$ & $\mathrm{G}$ & B & B & G & $\mathrm{G}$ \\
\hline 9 & G & B & G & B & G & B & B & G & G \\
\hline 10 & G & $\mathrm{G}, \mathrm{B}(1)^{3}$ & G & B & G & B & B & G & B \\
\hline 11 & G & $\mathrm{B}(7), \mathrm{G}(3)$ & G & B & $\mathrm{G}$ & B & B & G & B \\
\hline 12 & $\mathrm{~A}(9), \mathrm{B}(1)$ & $\mathrm{B}(8), \mathrm{A}(2)$ & B & B & B & $\mathrm{B}, \mathrm{A}$ & B & B & B \\
\hline 13 & B & B & B & B & B & B & B & B & B \\
\hline 14 & B & $\mathrm{B}(9), \mathrm{A}(1)$ & B & B & B & B & B & B & B \\
\hline 15 & B & B & B & B & B & B & B & B & B \\
\hline 16 & B & B & B & B & $\mathrm{G}, \mathrm{B}$ & B & B & $\mathrm{G}, \mathrm{A}$ & B \\
\hline 17 & G & B & $\mathrm{G}, \mathrm{B}$ & B & A & B & B & G & B \\
\hline 18 & $\mathrm{G}$ & B & $\mathrm{B}$ & B & A & B & B & $\mathrm{G}$ & B \\
\hline 19 & A & A & $\mathrm{B}$ & A & A & B & A & A & A \\
\hline 20 & A & A & A & A & A & A & A & A & A \\
\hline 21 & A & A & G & A & A & A & B & A & A \\
\hline 22 & A & A & A & A & A & A & A & A & A \\
\hline 23 & A & A & A & A & A & A & A & A & A \\
\hline 24 & A & A & A & A & A & A & A & A & A \\
\hline
\end{tabular}

${ }^{1}$ G: Gnaisse; B: Basalto; A:Arenito+sedimentos do terciário.

${ }^{2} \mathrm{C} 1, \mathrm{C} 2, \mathrm{C} 3$ e C4 são conjuntos de variáveis formados pelos teores de $\mathrm{Cd}, \mathrm{Cr}, \mathrm{Cu}, \mathrm{Fe}, \mathrm{Mn}, \mathrm{Ni}, \mathrm{Pb}$ e Zn, em mg/kg, extraídos pelos procedimentos de extração total com $\mathrm{HNO}_{3} / \mathrm{HClO}_{4}$ (4:1) (C1) e $\mathrm{HF} / \mathrm{HNO}_{3} / \mathrm{HClO}_{4}(20: 6: 3)(\mathrm{C} 2)$ na TFSA e $\mathrm{HF} / \mathrm{HNO}_{3} / \mathrm{HClO}_{4}$ (20:6:3) na argila (C3); o C4 é formado pelos três primeiros conjuntos, ou seja, os elementos extraídos com $\mathrm{HNO}_{3} / \mathrm{HClO}_{4}(4: 1)$ e $\mathrm{HF} / \mathrm{HNO}_{3} / \mathrm{HClO}_{4}(20: 6: 3)$ na TFSA e argila, respeitandose a disposição individual das variáveis em cada grupo: $\mathrm{C} 4=\mathrm{C} 1+\mathrm{C} 2+\mathrm{C} 3$.

${ }^{3}$ Casos em que as repetições não foram classificadas como de origem de um único material, sendo apresentada a segunda classificação e, entre parênteses, o número de repetições em que isto ocorreu. 
seu enquadramento e mesmo proximidade de solos de basalto. Porém, a análise discriminante, como técnica de adequação da classificação proposta, indicou a formação a partir do basalto. Finalmente, a partir do solo 19 constata-se a influência do arenito+sedimentos do terciário na formação dos solos, predominando, tanto na análise discriminante quanto nas demais técnicas utilizadas, a maior participação deste solo.

A classificação geral, exposta na Tabela 4, definida pela avaliação conjunta das análises de agrupamento e discriminante, se comparada com as individuais feitas com base nas funções discriminantes de Anderson, indica que entre os conjuntos de variáveis utilizados, a partição que mais se aproximou da geral proposta foi a obtida com o $\mathrm{C} 2$ na profundidade de $50-60 \mathrm{~cm}$, somente com divergência para o solo 8 . Tal fato torna evidente que, nas condições deste estudo, a extração dos metais pesados na TFSA e com uso de $\mathrm{HF} / \mathrm{HNO}_{3} / \mathrm{HClO}_{4}$, na proporção 20:6:3, parece ser a condição mais adequada para esse tipo de investigação, o mesmo podendo ser referido à profundidade de estudo, no caso de 50 a $60 \mathrm{~cm}$, até pelas próprias contribuições antrópicas possíveis de ocorrerem em superfície.

Deve-se ressaltar a importância do conhecimento prévio da área em estudo, através de levantamentos geológicos e mapas, bem como as idas a campo, no uso conjunto de técnicas mais sistematizadas, como as utilizadas, tornando a definição da origem dos diferentes solos coletados na topolitoseqüência estudada mais acertada, uma vez que dois desses solos estavam, apesar de apresentarem teores semelhantes, separados literalmente em distância, além de várias outras características bastante diferenciadas. Vários estudos indicam que os teores no solo dos elementos estudados refletem bem o material que lhes deram origem (Moura, 1985; Curi \& Franzmeier, 1987; Jing-Sheng et al., 1993; Pettry \& Switzer, 1993). Todavia, a proximidade numérica, quanto aos teores obtidos, pode conduzir a resultados que não possibilitem a diferenciação de um ou outro solo. De certa forma é possível que um maior número de pontos amostrados seja útil nesta diferenciação, porém, uma avaliação mais global é necessária, não podendo se ater somente à análise estatística, além de escolha adequada dos métodos de análise química a serem utilizados.
Poucos trabalhos existem na literatura referentes aos procedimentos adotados no presente estudo, principalmente quanto ao uso das técnicas multivariadas, o que leva a crer que o seu potencial de aplicação é elevado. Moura (1985) e Li et al. (1992) fizeram uso de análises de agrupamento e discriminante em estudos semelhantes, porém adotando técnicas diferenciadas das aqui utilizadas, obtendo-se resultados que atenderam aos objetivos propostos, porém, a partir de uma base de dados bastante superior à disponível para o trabalho em questão. Somente a análise discriminante proposta de Anderson (1958) não foi suficiente para definir claramente a partição proposta pelas demais técnicas, além de não haver concordância entre as discriminações feitas, quando se usam frações do solo e mesmo métodos químicos diferentes. Quanto a este último aspecto, deve-se ressaltar o fato de como um único procedimento pode levar a conclusões nem sempre confiáveis, o resultado final somente pôde ser obtido por uma avaliação conjunta de diferentes situações.

Os resultados obtidos indicam que as observações a campo e mesmo as informações de levantamentos diversos quando confrontadas podem ser questionadas quanto às possíveis inter-relações. Verifica-se que apesar de características ou propriedades (cor de solo, textura, teores de $\mathrm{Fe}_{2} \mathrm{O}_{3}$ ) indicarem a origem de determinado material, como é o caso dos solos 7, 8 e 9, na transição do gnaisse-basalto, e 19 e 24, originários de arenito+sedimentos do terciário, 19 a 24, a mesma pode não ser tão verdadeira. Constata-se, portanto, que os metais pesados exercem bem o papel de elementos diferenciadores dos materiais de origem estudados, definindo a intensidade de influência de um ou outro material na formação dos solos. Comparações entre a classificação geral obtida pelas análises de agrupamento e discriminante (Tabela 4) evidenciam bem tais observações, pois pode-se verificar a ocorrência de solos com classificação associada à origem basáltica (LR), enquanto pelo estudo proposto a sua origem está mais relacionada ao gnaisse, com as inferências pertinentes a essa origem.

Também deve ser salientado a relação dessas constatações com o status do solo em termos dos elementos estudados, tanto no que se refere ao 
potencial de incorporação na cadeia trófica pela sua maior abundância em algumas situações, como também escassez, a tendência de deficiência desses elementos em plantas cultivadas. Pelo que foi apresentado, as áreas definidas como originárias de arenito+sedimentos do terciário e gnaisse, situadas próximas à transição com o basalto, mas com fortes características ou propriedades visuais, químicas e físicas e mesmo mineralógicas, e teoricamente com teores de metais pesados em maior proporção, tenderão a apresentar deficiências desses elementos, o que nem sempre poderá ser percebido pelos técnicos envolvidos. Investigação de maior esclarecimento do status nutricional dos elementos em estudo, a partir de técnicas de agrupamento e discriminação, foi conduzido por Li et al. (1992), com bons resultados, porém, sem a preocupação de avaliação conjunta com o material de origem. reforçando o tipo de estudo apresentado.

\section{CONCLUSÕES}

1. Os metais pesados são indicativos do material de origem, podendo ser usados na delimitação da área de influência destes.

2. As técnicas multivariadas adotadas se mostram viáveis para este tipo de estudo, porém somente quando utilizadas conjuntamente.

\section{REFERÊNCIAS}

ANDERSON, T.W. An introduction to multivariate statistical analysis. New York: John Wiley, 1958. $374 \mathrm{p}$.

ANGELONE, M.; VASELLI, O.; BINI, C.; CORADOSSI, N. Pedogeochemical evolution and trace elements availability to plants in ophiolitic soils. The Science of the Total Environment, Amsterdam, v.129, p.291-309, 1993.

BITELL, J.; MILLER, R.J. Lead, cadmium and calcium selectivity coefficients on a montmorilonite, illite and kaolinite. Journal of Environment Quality, Madison, v.3, p.250-253, 1974.

BUSACCA, A.J.; SINGER, M.J. Pedogenesis of a chronosequence in the Sacramento Valley, California, U.S.A. II. Elemental chemistry of silt fractions. Geoderma, Amsterdam, v.44, p.43-75, 1989.
CHITTLEBOROUGH, D.J.; OADES, J.M.; WALKER, P.H. Textural diferentiation in chronosequences from eastern Australia. III. Evidence from elemental chemistry. Geoderma, Amsterdam, v.32, p.227-248, 1984.

CIAVATTA, C.; GOVI, M.; SIMONI, A.; SEQUI, P. Evaluation of heavy metals during stabilization of organic matter in compost produced with municipal solid wastes. Bioresources Technology, Essex, v.43, p.147-153, 1993.

CRUZ, C.D.; REGAZZI, A.J. Modelos biométricos aplicados ao melhoramento genético. Viçosa: UFV, 1994. 390p.

CURI, N. Lithosequence and toposequence of oxisols from Goiás and Minas Gerais States, Brazil. West Lafayette: Purdue Univ., 1983. 158p. Ph.D. Thesis.

CURI, N.; FRANZMEIER, D.P. Effect of parent rocks on chemical and mineralogical properties of some Oxisols in Brazil. Soil Science Society of American. Journal, Madison, v.51, p.153-158, 1987.

DEFELIPO, B.V.; RIBEIRO, A.C. Análise química do solo. Viçosa: UFV, 1981. 17p. (Boletim de Extensão, 29).

EMBRAPA. Serviço Nacional de Levantamento e Conservação de Solos (Rio de Janeiro, RJ). Manual de métodos de análise de solo. Rio de Janeiro, 1979. Não paginado

ESSER, K.B.; BOCKHEIM, J.G.; HELMKE, P.A. Trace element distribution in soils formed in the Indiana dunes, U.S.A. Soil Science, Baltimore, v.152, n.5, p.340-350, 1991.

FERREIRA, S.A.D.; SANTANA, D.P.; FABRIS, J.D.; CURI, C.; NUNES FILHO, E.; COEY, J.M.D. Relações entre magnetização, elementos traços e litologia de duas sequiências de solos do Estado de Minas Gerais. Revista Brasileira de Ciência do Solo, Campinas, v.18, n.2, p.167-174, 1994.

GAMBRELL, R.P.; WIESEPAPE, J.B.; PATRICK JUNIOR, W.H.; DUFF, M.C. The effects of $\mathrm{pH}$, redox and salinity on metal release from a contaminated sediment. Water, Air and Soil Pollution, Dordrecht, v.57/58, p.359-367, 1991.

Pesq. agropec. bras., Brasília, v.34, n.8, p.1451-1465, ago. 1999 
GUPTA, S.K.; STADELMANN, F.X. Effect of sewage sludge on the biorelevant cadmium concentration. In : IN TER N AT I ON A L S Y MPOS I UM PROCESSING AND USE OF SEWAGE SLUDGE, 3., 1983, Brighton. Proceedings... Brighton: Commission of the European Communities, 1983. p. $435-445$

JENG, A.S.; BERGSETH, H. Chemical and mineralogical properties of norwegian alum shale soils, with special emphasis on heavy metal content and availability. Acta Agricultural Scandinavica, Section B, Soil and Plant Science, Copenhagen, v.42, p.88-93, 1992.

JING-SHENG，C.; BAO-SHAN，D.; MAO, P.; XUE-JUN, W.; SHUI-QUAN, Z.; QUN, HE. Geographical tendencies of trace element contents in soils derived from granite, basalt and limestone of Eastern China. Pedosphere, Beijing, v.3, n.1, p.45-55, 1993.

KRAUSKOPF, K.B. Geochemistry of nutrients. In: MORTVEDT, J.J.; GIORDANO, P.M.; LINDSAY, W.M. (Eds.). Micronutrients in agriculture. Madison: Soil Science Society of America, 1972. p.7-40.

LAGERWERFF, J.V. Lead, mercury and cadmium as environmental contaminants. In: DINAUER, R.C. (Ed.). Micronutrients in agriculture. Madison: Soil Science Society of America, 1977. p.593-628.

LEMOS, R.C.; SANTOS, R.D. Manual de descrição e coleta de solo no campo. Campinas: SBCS/ Embrapa-SNLCS, 1984. 45p.

LI, G.C.; MAHLER, R.L.; EVERSON, D.O. Micronutrients in the Kootenai River Valley of Northern Idaho. II. Use of cluster and discriminant analyses to evaluate soil micronutrient status. Communications in Soil Science and Plant Analysis, New York, v.23, n.11/12, p.1179-1194, 1992.

MAHALANOBIS, P.C. On the generalized distance in statistic. Proceedings of National Institute of Sciences of India, v.2, p.49-55, 1936.

MALMER, N.; HORTON, D.G.; VITT, D.H. Element concentrations in mosses and surface waters of western canadian mires to precipitation chemistry and hydrology. Ecography, Copenhagen, v.15, p.114-128, 1992.
MANN, S.S.; RITCHIE, G.S.P. The influence of $\mathrm{pH}$ on the forms of cadmiun in four west Australian soils. Australian Journal of Soil Research, Melbourne, v.31, p.255-270, 1993.

MARIÑO, F.; LIGERO, A.; DIAZ COSIN, D.J. Heavy metals and earthworms on the border of a road next to Santiago (Galicia, northwest of Spain). Initial results. Soil Biology and Biochemistry, Oxford, v.24, n.12, p.1705-1709, 1992.

MINAS GERAIS. Secretaria de Planejamento e Coordenadoria Geral. Instituto de Geociências Aplicadas. Mapa geológico do Estado de Minas Gerais. Belo Horizonte, 1976. Mapa color, 120x100cm. Projeção policônica. Escala 1:1.000.000.

MITCHELL, R.L. Trace elements in soils. In: BEAR, F.E. (Ed.). Chemistry of the soil. New York: Reinhold, 1964. p.320-368.

MOURA, C.A.V. Aplicação de tratamento estatístico multivariante em dados geoquímicos de solo no mapeamento geológico na província de Carajás (alvo 2 - corpo 4). Revista Brasileira de Geociências, v.15, n.3, p.241-248, 1985.

MUNSELL COLOR. Munsell soil color charts. Baltimore: Macbeth Division of Kollmorgen Corporation, 1975. Não paginado.

PASCOE, G.A.; BLANCHET, R.J.; LINDER, G. Bioavailability of metals and arsenic to small mammals at a mining waste-contaminated wetland. Archives of Environment Contaminations and Toxicology, New York, v.27, p.44-50, 1994.

PETTRY, D.E.; SWITZER, R.E. Heavy metal concentration in selected soils and parent materials in Mississippi. Meridian: MSU, 1993. 33p. (MSU Bulletin, 998).

PICCOLO, A.; CELANO, G. Distribution of heavy metals in profiles of a hydromorphic soil system. Fresenius Environment Bulletin, Basel, v.1, p.16-21, 1992.

PLICHTA, W.; KUCZYNSKA, I. Metal contents in soils of Kaffiöyra, Spitsbergen. Polish Polar Research v.12, n.2, p.183-193, 1991.

RAO, R.C. Advanced statistical methods in biometric research. New York: John Wiley, 1952. 390p. 
RESENDE, M.; ALLAN, J.; COEY, J.M.D. The magnetic soils of Brazil. Earth Planetary Science Letters, v.78, p.322-326, 1986.

SHOTYK, W.; NESBITT, W.; FYFE, W.S. Natural and anthropogenic enrichments of trace metals in peat profiles. International Journal Coal Geology, Amsterdam, v.20, p.49-84, 1992.

SINGH, M.; SEKHON, G.S. DTPA extractable micronutrient cations in twenty soil series of India. Journal of the Indian Society of Soil Science, New Delhi, v.39, p.129-133, 1991.

TAYLOR, R.W.; IBEABUCHI, I.O.; SISTANI, K.R.; SHUFORD, J.W. Heavy metal concentration in forage grasses and extractability from some acid mine spoils. Water, Air and Soil Pollution, Dordrecht, v.68, p.363-372, 1993.
TILLER, K.G. Micronutrients. In: COMMOWEALTH SCENTIFIC AND INDUSTRIAL RESEARCH ORGANIZATION. Soils, an australian viewpoint. London, Melbourne: Academic, 1980. p.365-388.

VALADARES, J.M.A.S. Cobre em solos do Estado de São Paulo. I. Cobre total. Bragantia, Campinas, v.34, n.4, p.125-132, 1975.

VALADARES, J.M.A.S.; CATANI, R.A. Zinco em solos do Estado de São Paulo. Bragantia, Campinas, v.34, n.5, p.133-139, 1975.

XING, B.; DUDAS, M.J. Trace and rare earth element content of white clay soils of the Three River Plain, Heilongjiang Province, P.R. China. Geoderma, Amsterdam, v.58, p.181-199, 1993. 